\title{
Entwicklung eines koaxialen Faltenbalgzylinders zur Übertragung von linearen Bewegungen ins Hochvakuum
}

\author{
Karin Siemroth
}

\section{Zusammenfassung}

In Zusammenarbeit zwischen der Technischen Fachhochschule Wildau und der Fa. Arc Precision GmbH Wildau wurde eine neue technische Lösung - der so genannte Koaxiale Faltenbalgzylinder - entwickelt, mit dem Linearbewegungen in Bereiche mit Hochvakuum übertragen werden können. Die Idee wurde zum Patent angemeldet, konstruiert, erprobt und fand Verwendung in einer Plasmaquelle der Fa. Arc Precision. Gegenüber bekannten Serienlösungen zeichnet sich der Koaxiale Faltenbalgzylinder durch kompakte und Platz sparende Bauweise, leichte Ansteuerbarkeit, Leckfreiheit, schnelle Bewegungen, gute axiale Führungsgenauigkeit, große übertragbare Kräfte sowie letztlich auch durch Kosteneinsparungen aus. Am Beispiel der Entwicklung des Koaxialen Faltenbalgzylinders werden allgemeine Methoden beim Lösen von Entwicklungsaufgaben in enger Verbindung mit der Lehre dargestellt.

\begin{abstract}
In cooperation between the University of Applied Sciences Wildau and the company Arc Precision GmbH Wildau a new solution - named as »coaxial bellows cylinder « - to bring linear movements into a vacuum chamber was found. The new solution was patented, designed, tested and finally used in a plasma source of the Arc Precision company. In comparison to well-known series products, the coaxial bellows cylinder is much more compact, easy to control, fast, is free of leakages, good axial guide precision and large forces can be transferred - and last but not least, is a cost saving solution. Common methods of engineering design process in close contact to the teaching are exemplified using the development of the coaxial bellows cylinder.
\end{abstract}

\section{$1 \quad$ Einleitung}

Die Reife technischer Produkte ist im hohen Maße davon abhängig, in welchen Stückzahlen diese zum Einsatz gelangen. Es muss deshalb nicht verwundern, wenn einerseits am Markt technisch und technologisch ausgefeilte Produkte sehr preiswert verfügbar sind, andererseits sich für die unterschiedlichsten Anwendungen jenseits von Großserien immer wieder aufwendige und wenig optimierte Konstruktionen offenbar recht stabil etabliert haben.

Vakuumanwendungen waren lange Zeit einem kleinen Anwenderklientel vorbehalten, das aus dem Forschungsbereich oder dem Hightechbereich stammt. Da hier ganz besonderer Wert auf hohe Qualität gelegt wird, werden in der Regel auch die für üblich und normal gehaltenen Preise bezahlt.

So werden z. B. für die Übertragung linearer Bewegungen ins Hochvakuum teilweise sehr aufwendige und kostenintensive Konzepte angewendet, die sich zwar bei genauerer Analyse alles andere als technisch ausgefeilt erweisen, aber dennoch ausgezeichnet am Markt behaupten können. Es lohnte sich, für diese Hochvakuumbauteile methodisch nach neuen Konzepten zu suchen. So entstand die Idee des Koaxialen Faltebalgzylinders, der inzwischen gebaut, erfolgreich getestet und als Patent angemeldet wurde.

\section{Aufgabenstellung}

Für eine industrielle Vakuumbeschichtungsanlage zur Herstellung von superharten Kohlenstoffschichten ist für die kontinuierliche Arbeit der Anlage ein automatisches Nachladen von Graphittargets in den Arbeitsbereich eines Plasmaverdampfers zu gewährleisten, ohne den Beschichtungsprozess zu stören oder anzuhalten. Dafür wurde ein automatischer Targetwechsler entwickelt, in dem zwei Stempel linear zu bewegen sind, die folgenden Anforderungen gerecht werden müssen:

- Hub ca. $40 \mathrm{~mm}$ mit definierten Endlagen

- lichte Weite für Stempel > $60 \mathrm{~mm}$

- Verfahrzeit < $1 \mathrm{~s}$

- Takt ca. 10 min

- Übertragung leckfrei ins Hochvakuum

- Stromstärke > $2 \mathrm{kA}$

- elektrische Isolierung gegenüber dem Gehäuse

- Wasserkühlung

- Anpresskraft von ca. $700 \mathrm{~N}$ halten

- axiale Führungsgenauigkeit < 0,5 mm

- Der verfügbare Bauraum ist extrem begrenzt. 


\section{Kataloglösungen}

In den einschlägigen Katalogen für Vakuumanwendungen finden sich verschiedene Lösungen, um Linearbewegungen im Vakuum zu realisieren.

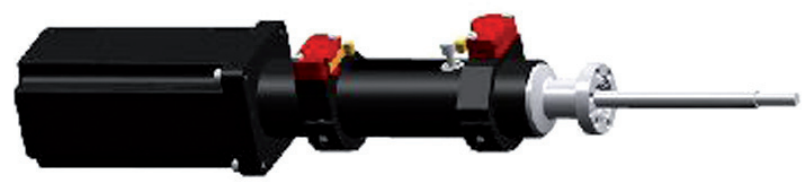

Abb. 1: Lineardurchführung mit Motor bis NW 40 nach [3]

Abb. 1 zeigt eine Lineardurchführung, bei der zunächst eine Drehbewegung initiiert, ins Vakuum übertragen und dort in eine Längsbewegung gewandelt wird. Solche Lineardurchführungen sind nur bis zu Nennweiten von $40 \mathrm{~mm}$ verfügbar. Für die aktuelle Aufgabe ist diese Variante aus folgen Gründen unbrauchbar:

- Die vorhandenen Strom- und Kühlwasserleitungen können nicht integriert werden.

- Der Durchmesser des Stempels ist zu groß, damit muss der gesamte Hub innerhalb der Vakuumkammer als Platzbedarf bereitgestellt werden.

- Die erforderliche Anpresskraft wird nicht erreicht.

- Die Bewegung ist zu langsam.

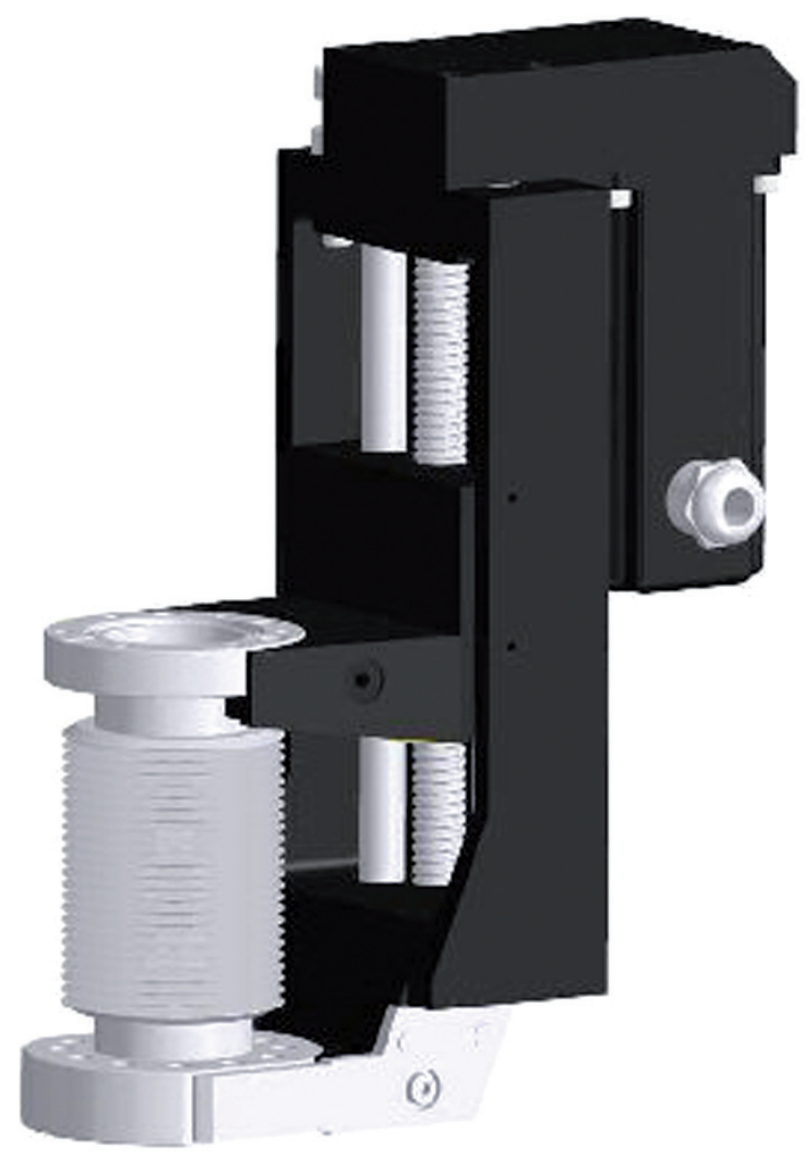

Abb. 2: Präzisions UHV-Lineardurchführung nach [3]

Bei der Lineardurchführung nach Abb. 2 erzeugt ein Motor ein Drehmoment. Dieses wird mit einer Getriebestufe übersetzt und mittels Spindel und Spindelmutter in eine Längskraft gewandelt. So kann die erforderliche Kraft aufgebracht werden, die für die Bewegung gegen den atmosphärischen Druck benötigt wird. Die lineare Führung übernehmen zwei Säulen, die in der Ebene der Spindel angeordnet sind. Die Kräfte werden über Kragträger geleitet, die auf Grund der Präzisionsanforderungen sehr verformungsstabil ausgebildet sind. Ein geschweißter Faltenbalg kann sich im Rahmen seines ausgelegten Hubes reversibel verformen und gleichzeitig die Vakuumkammer gegen den Außenbereich leckfrei abdichten.

Diese Lineardurchführung bietet zwar die Anschlussmöglichkeit für Strom und Wasser, ist für den konkreten Fall aber nicht einsetzbar, da die Verfahrgeschwindigkeit viel zu gering ist und der notwendige Bauraum nicht zur Verfügung steht.

\section{Methodik der Lösungsfindung}

Für die methodische Entwicklung neuer Lösungskonzepte für innovative Produkte bietet die VDI-Richtlinie 2221 folgenden Ansatz [1]. Nach Klärung der Aufgabe ist zunächst die Funktionsstruktur zu analysieren. Für die unterschiedlichen Einzelfunktionen wird nach neuen Teilkonzepten gesucht und diese in einem morphologischen Kasten neu verknüpft (siehe Tab. 1). In einem weiteren Schritt können weitere Einzelfunktionen definiert werden, die dann neu kombiniert eine veränderte Funktionsstruktur aufweisen. Aus der so entstandenen Lösungsvielfalt können nun geeignete Lösungen ausgewählt werden.

\begin{tabular}{|c|c|c|c|c|c|}
\hline Teilfunktion & Lösungsvariar & ten & & & \\
\hline Antrieb & von Hand & elektrisch & $\begin{array}{l}\text { pneu- } \\
\text { matisch }\end{array}$ & $\begin{array}{l}\text { hydrau- } \\
\text { lisch }\end{array}$ & \\
\hline $\begin{array}{l}\text { Bewegungs- } \\
\text { hub }\end{array}$ & Zylinder & Spindel & Zahnstange & $\begin{array}{l}\text { Linear- } \\
\text { motor }\end{array}$ & $\begin{array}{l}\text { Piezo- } \\
\text { element }\end{array}$ \\
\hline Steuerung & def. Endlage & Sensor & $\begin{array}{l}\text { verstellbarer } \\
\text { Anschlag }\end{array}$ & & \\
\hline $\begin{array}{l}\text { Aufnahme } \\
\text { für Strom- } \\
\text { leitungen/ } \\
\text { Wasser- } \\
\text { kühlung }\end{array}$ & $\begin{array}{l}\text { integriert in } \\
\text { Flansch }\end{array}$ & $\begin{array}{l}\text { separat, } \\
\text { flexible Ver- } \\
\text { bindungen }\end{array}$ & & & \\
\hline $\begin{array}{l}\text { axiale } \\
\text { Führung }\end{array}$ & $\begin{array}{l}\text { exzentrische } \\
\text { Säulen- } \\
\text { führung } \\
\end{array}$ & $\begin{array}{l}\text { zentrische } \\
\text { Säulen- } \\
\text { führung }\end{array}$ & $\begin{array}{l}\text { Schwalben- } \\
\text { schwanz- } \\
\text { führung }\end{array}$ & \begin{tabular}{|l} 
koaxiale \\
Führung
\end{tabular} & \\
\hline Zentrierung & $\begin{array}{l}\text { hohe } \\
\text { Führungs- } \\
\text { genauigkeit }\end{array}$ & $\begin{array}{l}\text { geringe } \\
\text { Bauteilver- } \\
\text { formungen }\end{array}$ & \begin{tabular}{|l} 
selbst- \\
zentriert
\end{tabular} & & \\
\hline $\begin{array}{l}\text { leckfreie } \\
\text { Abdichtung }\end{array}$ & $\begin{array}{l}\text { Schiebe- } \\
\text { durch- } \\
\text { führung }\end{array}$ & $\begin{array}{l}\text { alle Bauteile } \\
\text { im Vakuum }\end{array}$ & Faltenbalg & $\begin{array}{l}\text { magne- } \\
\text { tische } \\
\text { Kraftüber- } \\
\text { tragung }\end{array}$ & $\begin{array}{l}\text { Schutz- } \\
\text { gas mit } \\
\text { großen } \\
\text { Molekü- } \\
\text { len }\end{array}$ \\
\hline $\begin{array}{l}\text { Anpress- } \\
\text { druck für } \\
\text { den Stempel }\end{array}$ & $\begin{array}{l}\text { unmittelbar } \\
\text { vom Antrieb }\end{array}$ & $\begin{array}{l}\text { mittelbar } \\
\text { vom Antrieb }\end{array}$ & $\begin{array}{l}\text { mittels } \\
\text { Atmosphä- } \\
\text { rendruck }\end{array}$ & & \\
\hline $\begin{array}{l}\text { Verbindung } \\
\text { zur Vakuum- } \\
\text { kammer }\end{array}$ & Normflansch & $\begin{array}{l}\text { Spezial- } \\
\text { flansch }\end{array}$ & $\begin{array}{l}\text { Schweißver- } \\
\text { bindung }\end{array}$ & & \\
\hline $\begin{array}{l}\text { Platz für ein- } \\
\text { gefahrenen } \\
\text { Stempel }\end{array}$ & $\begin{array}{l}\text { verbleibt in } \\
\text { der Vakuum- } \\
\text { kammer }\end{array}$ & $\begin{array}{l}\text { lichte Weite } \\
\text { in der Durch- } \\
\text { führung }\end{array}$ & & & \\
\hline
\end{tabular}

Tab. 1: Morphologischer Kasten 
Neben einer Vielzahl möglicher Kombinationen für die Übertragung von linearen Bewegungen ins Vakuum sind hier nur zwei herausgehoben. Die grau untersetzte Kombination stellt die in Vakuumkatalogen verfügbare Lineardurchführung nach Abb. 2 dar. Stark eingerahmt ergibt sich der Koaxiale Faltenbalgzylinder.

Nachdem für die gestellte Aufgabe mit dem Koaxialen Faltenbalgzylinder bereits eine geeignete Lösung entwickelt war, erschien die Aufgabe so interessant und vielschichtig, dass sie den Studenten des Studienganges Maschinenbau im 4. Semester noch einmal als Semesteraufgabe im Lehrgebiet Produktentwicklung gestellt wurde. Bei dieser allerdings etwas vereinfachten Aufgabenstellung sollte die vorhandene Lineardurchführung nach Abb. 2 konstruktiv verbessert werden. Eine Reihe der in Tab. 1 aufgezeigten kreativen Lösungsvorschläge für die Erfüllung der Teilfunktionen und die Neugestaltung der Funktionsstruktur entstanden in den Übungen der Seminargruppe M/05. Es zeigte sich, dass auch auf den ersten Blick ausgefallene Ideen die Diskussion voranbringen und damit hilfreich sein können, denn zu frühes Abwerten von Ideen hemmt den kreativen Prozess.

\section{Der Koaxiale Faltenbalgzylinder}

Eine mögliche Lösung für die Übertragung der linearen Bewegung ins Vakuum nach Tab. 1 ist der Koaxiale Faltenbalgzylinder. Diese Konstruktion entstand bei der Entwicklung einer Wechslereinrichtung für die Firma Arc Precision GmbH Wildau und erfüllt die gestellten Anforderungen vollumfänglich.

Der Grundgedanke dieser Konstruktion ist es, die Kraft des atmosphärischen Luftdrucks auszunutzen, die bereits Otto von Guericke bei seinen Versuchen mit den Magdeburger Halbkugeln faszinierte. So wird ein Deckel vom Durchmesser d durch den Atmosphärendruck $\mathrm{p}_{\mathrm{A}}$ mit der Kraft F gegen eine Vakuumkammer gedrückt, in der ein Unterdruck $\mathrm{p}_{\mathrm{V}}$ herrscht:

$$
\mathrm{F}=\left(\mathrm{p}_{\mathrm{A}}-\mathrm{p}_{\mathrm{V}}\right) \pi \mathrm{d}^{2} / 4 \quad \begin{aligned}
& \mathrm{p}_{\mathrm{A}}=10^{5} \mathrm{~Pa}, 1 \text { bar } \\
& (\text { atmosphärischer Druck) } \\
& \\
& \mathrm{p}_{\mathrm{V}}=10^{-4} \mathrm{~Pa} \\
& \text { (Druck im Hochvakuum) }
\end{aligned}
$$

Diese Kraft wird genutzt, die Bauteile in Richtung des Vakuums zu bewegen und in der Vakuumkammer mit einer definierten Haltekraft zu spannen. Zum Lösen wird eine Gegenkraft gleicher Größe benötigt. Diese Kraft wird in einem Zylinder erzeugt, der außen koaxial um den Faltenbalg angeordnet und damit optimal Platz sparend ist. Abb. 1 zeigt die konstruktive Umsetzung dieser Idee.

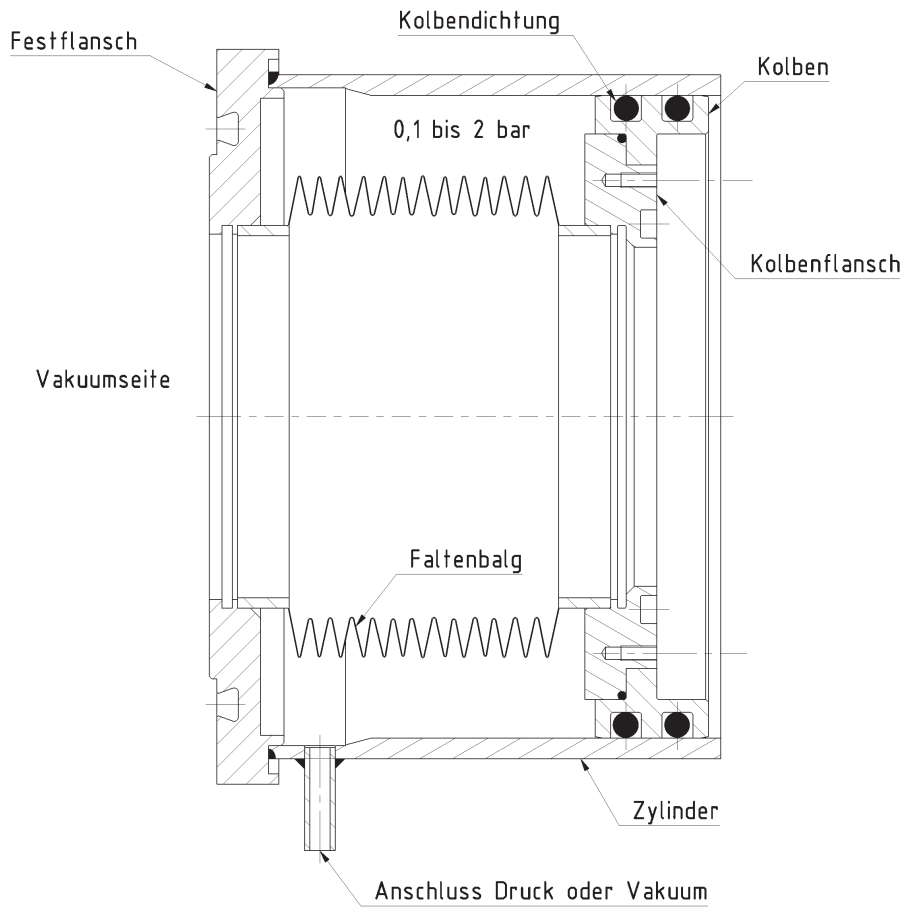

Abb. 3: Koaxialer Faltenbalgzylinder

Im Folgenden wird die Funktion im Detail erläutert.

- Der Koaxiale Faltenbalgzylinder wird mit dem Festflansch an die Vakuumkammer angeflanscht.

- In den Kolbenflansch wird im konkreten Anwendungsfall der Stempel oder wahlweise ein beliebiger Flansch eingesetzt.

- Zwischen Festflansch und Kolbenflansch ist ein metallischer Faltenbalg eingeschweißt, der in Längsrichtung sehr flexibel ist, dem Druckunterschied aber gut standhalten kann. Solche Bälge sind Standard in der Vakuumtechnik.

- Mit dem Kolbenflansch verbunden ist der Kolben mit den Kolbendichtungen.

- Die Kolbendichtungen gleiten an der Innenwand des Zylinders und dichten den Druckraum so gut gegen Druckverluste bzw. Leckverluste ab, dass selbst ein Unterdruck von ca. 0,1 bar über längere Zeit gehalten wird.

- Der Kolben bewegt sich im Zylinder in Abhängigkeit von den Druckverhältnissen, die über eine Anschlussleitung an Druckluft oder Vakuum verändert werden.

- Im einfachen Fall bewegt sich der Kolbenflansch nur durch die Erzeugung von Vakuum in der Kammer nach links und spannt mit der einfachen Kraft, die der Luftdruck über den Durchmesser des Faltenbalges aufbaut.

- Zusätzlich kann der Druckraum im Zylinder mit Vorvakuum beaufschlagt werde. Dadurch wird die Spannkraft etwa doppelt so groß.

- Erhält der Druckraum im Zylinder einen Überdruck bis etwa 2 bar, so bewegt sich der Kolbenflansch nach rechts.

- Die Endlagen der Bewegung sind durch Anschläge einstellbar. 
Der Koaxiale Faltenbalgzylinder hat entscheidende Vorteile gegenüber der Lineareinheit nach Abb. 2:

- Sehr kompakte und Platz sparende Leichtbaukonstruktion.

- Schnelle Bewegung möglich.

- Die Ansteuerung erfolgt über ein Ventil, welches zwischen Druck und Vorvakuum umschaltet.

- Der Kolben im Zylinder gewährleistet eine gute axiale Führungsgenauigkeit für den Stempel.

- Große Kräfte übertragbar, hohe Spannkraft realisierbar.

- Trotz Einzelanfertigung ergeben sich bereits Kosteneinsparungen gegenüber der Serienlösung.

Ein erster Prototyp wurde im Dezember 2006 angefertigt. Hier zeigten sich neben der grundsätzlichen Machbarkeit aber auch deutliche Schwächen. Der Kolben ließ sich zu schwer bewegen und eine Demontagemöglichkeit fehlte. Die überarbeitete Variante erhielt einen geteilten Kolben mit angepassten Werkstoffpaarungen und optimierten Passungen. Weiterhin wurden die Abmessungen noch einmal verringert. Diese Koaxialen Faltenbälge wurde in der Firma Arc Precision inzwischen ausgiebig getestet und erfüllen ihre Aufgaben hervorragend. Über die Zukunftsagentur Brainshell wurde der Koaxiale Faltenbalgzylinder inzwischen als Europäische Patentanmeldung eingereicht [2].

\section{Ausblick}

Die konstruktive Weiterentwicklung des Koaxialen Faltenbalgzylinders zu einer Baureihe mit verschiedenen Nennweiten und Hüben sowie verschiedenen Normflanschanschlüssen wird der nächste Schritt zur Serienreife und industriellen Vermarktung sein. Diese Aufgabe wird die Technische Fachhochschule zusammen mit einem Industriepartner aus der Vakuumbranche bearbeiten, der auch die spätere Vermarktung realisieren kann. Im Fall der Patenterteilung und industriellen Vermarktung werden für die TFH Wildau Lizenzeinnahmen erwartet.

\section{Danksagung}

Mein besonderer Dank gilt der Firma Arc Precision GmbH Wildau, die nicht nur die anspruchsvolle Aufgabe stellte, sondern auch die materiellen Mittel zum Bau und Test von Funktionsmustern bereitstellte und mit wertvollen Hinweisen zum Gelingen der Aufgabe beitrug.

\section{Referenzen}

[1] Pahl, Beitz, Konstruktionslehre, Springer Verlag Heidelberg 2005

[2] Patentanmeldung P5I90EP00, eingereicht am 04.07.2007

[3] VAb: UHV Katalog 2004/2005, 25337 Elmshorn

\section{Literatur}

Pahl, Beitz, Konstruktionslehre, Springer Verlag Heidelberg 2005

Patentanmeldung P5I90EP00, eingereicht am 04.07.2007

VAb: UHV Katalog 2004/2005, 25337 Elmshorn

\section{Autorin}

Prof. Dipl.-Ing. Karin Siemroth

Technische Fachhochschule Wildau

FB Ingenieurwesen/Wirtschaftsingenieurwesen

Labor für Konstruktionstechnik

Tel. +49 3375 508-231

karin.siemroth@tfh-wildau.de 